\title{
REVIEWS
}

\section{Genetic Factors in Selected Complex Congenital Malformations with Cleft Defect}

\author{
${ }^{1}$ Department of Plastic Surgery, Wroclaw Medical University, Polanica-Zdrój, Poland \\ ${ }^{2}$ Department of Plastic Surgery, Specialist Medical Center, Polanica-Zdrój, Poland
}

A - research concept and design; B - collection and/or assembly of data; C - data analysis and interpretation;

$\mathbf{D}$ - writing the article; $\mathbf{E}$ - critical revision of the article; $\mathbf{F}$ - final approval of article

\begin{abstract}
Cleft lips, alveolar ridges and palates are among the most common birth defects. There are over 500 different complex genetic disorders that include cleft defects. The most common related defects include abnormalities of the skeleton, skull, cardiovascular and nervous system. The occurrence of a cleft results from the interplay of multiple genes and environmental factors. Several thousand different mutations responsible for these syndromes have been discovered, whereas there are still numerous phenotypic cases of unknown genetic origin. The aim of this study was to present various clinical aspects and the latest discoveries with regard to genetic research in complex malformations, such as Van der Woude syndrome, popliteal pterygium syndrome, EEC syndrome, Pierre Robin sequence, various forms of Stickler syndrome, and Treacher Collins syndrome. These complex syndromes have different incidences, and most of them also have allelic variants with characteristic severities that differ even among close relatives. Easier access to genetic counseling and the lower cost of DNA testing in recent years can lead to new findings on the causes of such syndromes (Adv Clin Exp Med 2016, 25, 5, 977-987).
\end{abstract}

Key words: Treacher Collins syndrome, cleft palate, cleft lip, Pierre Robin syndrome, Van der Woude syndrome.

Cleft lips, alveolar ridges and palates are among the most common birth defects. Annually, more than 700 children are born in Poland and about 135,000 worldwide with these developmental disorders. Their frequency depends on race, gender and socioeconomic conditions. They occur most often among the Japanese (2.1 per 1000 births) and Chinese (1.7 per 1000) populations. In the Caucasian population the incidence rate is about 1.43 per 1000 births and in the black population 0.3 per 1000 births.

Clefts of the lip with/without concomitant cleft palate $(\mathrm{CL} / \mathrm{P})$ are about twice as common among boys, whereas isolated cleft palate $(\mathrm{CP})$ prevails in girls $(1.5: 1)$. Among unilateral cleft lip, a $2: 1$ predominance is seen for defects involving the left side, which is explained by the sequence of embryological development of the facial skeleton [1].

The distinct embryological and pathophysiological development mechanisms of isolated cleft lip and cleft palate is reflected in the division into clefts of the primary and secondary palate.
The primary palate consists of a lip, alveolar ridge of the jaw, and a part of the hard palate located at the front of the incisive foramen. The secondary palate involves a hard palate located at the back of the incisive foramen and the whole soft palate. Thirty percent of cases of cleft lip with cleft palate are complex congenital malformations with other coexisting malformations $[2,3]$. In the group with isolated cleft palate, the percentage of complex developmental defects is even higher, approaching $50 \%$ of all CP cases [4]. The most common related defects include abnormalities of the skeleton, skull, cardiovascular system, and nervous system.

Reconstruction treatment for children begins in infancy with lip and palate treatment at 6-18 months of age. The first descriptions of cleft lip operations come from ancient China. The annals of the Chinese dynasty in 390 BC contain a description of cleft surgery sutures on an 18-year-old man [5]. The first accurate medical descriptions of cleft palate reconstruction come from 1817 [6]. 
The milestones for today's surgical methods are the works of von Langenbeck (1861) [7] and of Veau (1931) [8] whose surgical techniques are used, with some modifications, to this day.

The occurrence of cleft results from the effects of multiple genes and from environmental factors. Its occurrence is highly dependent on individual characteristics; however, in patients with isolated cleft palate and those with cleft lip and palate, the most constant and specific risk factor remains a positive family history. The most important factor in the course of embryogenesis is an uneventful pregnancy, especially during the first trimester. The so-called environmental factors in this particular time may affect the process that contributes to cleft formation. The group of maternal risk factors include: smoking cigarettes, drinking alcohol and caffeine, using benzodiazepines, corticosteroids, certain antibiotics, antiepileptic drugs, exposure to organic solvents and pesticides, and nutritional factors (particularly vitamin supplementation). Clefts occur more frequently in families with low social status and poor living conditions. Moreover, it appears that the number and course of previous pregnancies and abortions, the age of parents, their occupation, and place of residence may affect the occurrence of the defect.

However, there is currently not enough data about a number of substances to allow us to unambiguously determine their role in the development or prevention of cleft formation. Folic acid administered before conception and during the first trimester is acknowledged as preventing neural tube birth defects. In craniofacial cleft defects, the effectiveness of this supplementation is not so clear. Until recently, folic acid was considered a preventive factor, but more recent meta-analyses that include the results of several prospective studies do not show strong evidence for the protective effect of folic acid alone. However, it is believed that a combination of folic acid with other multivitamin supplements may have such an effect [9]. There are also many doubts related to the effect of vitamin A and other retinoids. Although vitamin A in high doses is recognized as a teratogenic factor, according to an individual study in the Scandinavian countries, vitamin A appears to have a protective effect against $\mathrm{CP}$ formation; the effect on $\mathrm{CL} / \mathrm{P}$ was, however, inconclusive [10]. A more recent study on the Thai population confirmed the existence of a positive correlation between the intake of multivitamins and the reduced risk of cleft defects. The same effect is observed with meals containing liver, while the intake of menstrual cycle regulators by the mother was associated with an increased risk of offspring born with birth defects [11]. According to some studies, the occurrence of diseases like cold and fever in early pregnancy may also contribute to the formation of cleft anomaly [12].

\section{Complex Congenital Malformations with Cleft Defect}

Cleft defect is associated with over 500 different complex genetic disorders such as Van der Woude syndrome, popliteal pterygium syndrome, Roberts syndrome, Gorlin syndrome, EEC syndrome, CHARGE syndrome, Stickler syndrome, Miller syndrome, Kallmann syndrome, SmithLemli-Opitz syndrome, Pierre Robin sequence, Larsen syndrome, Bamforth-Lazarus syndrome, Kabuki syndrome, Cornelia de Lange syndrome, Treacher Collins syndrome, Andersen syndrome, Loeys-Dietz syndrome, Saethre-Chotzen syndrome, craniofrontonasal syndrome, holoprosencephaly, hydrolethalus, mental retardation associated with chromosome X syndrome, DiGeorge syndrome, and many others (see online Mendelian Inheritance in Man - OMIM). Recent genetic research has broadened our knowledge of birth developmental defects in complex malformations such as Van der Woude syndrome, popliteal pterygium syndrome, EEC syndrome, Pierre Robin sequence, various forms of Stickler syndrome, and Treacher Collins syndrome.

\section{Van der Woude Syndrome (VWS, OMIM 119300) and Popliteal Pterygium Syndrome (PPS, OMIM 119500)}

Van der Woude syndrome is one of the most common complex defects involving a cleft defect. It occurs with a frequency of $1: 35000-1: 100000$ and constitutes about $2 \%$ of all cleft defects. It was first described by Demarquay in 1845; the first extensive study of the syndrome was conducted by Anne Van der Woude in 1954, after whom this complex group of disorders was named [13]. Its characteristic features include salivary fistulas on both sides located medially, mostly within the lower lip with coexisting cleft lip with/without cleft palate (Fig. 1). The salivary fistulas occur and vary in severity from isolated cavities and sinuses to deep channels connected with secreting salivary glands. Fistulas often penetrate into the orbicularis oris muscle with salivary tanks located under the mucosa of the mouth. Other commonly related defects include dental agenesis (hypodontia) with loss of the lateral incisors in the maxilla and second premolar of the mandible and max- 


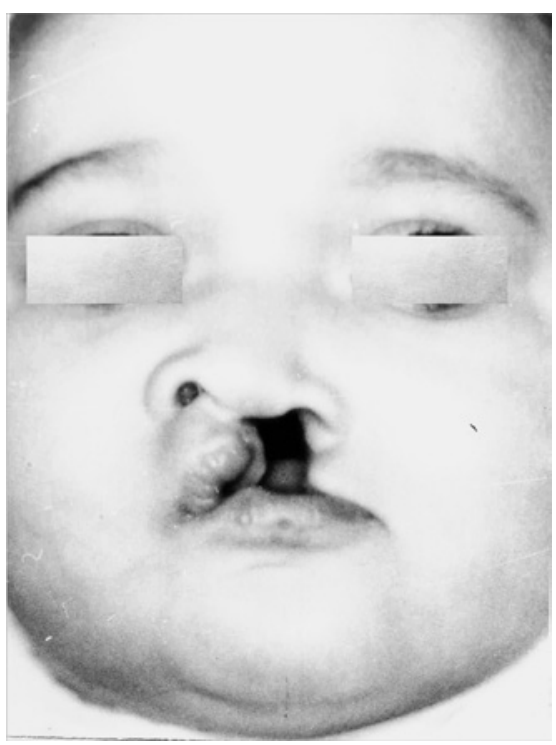

Fig. 1 A. A child with Van der Woude syndrome (VWS). Cleft lip, alveolus and palate

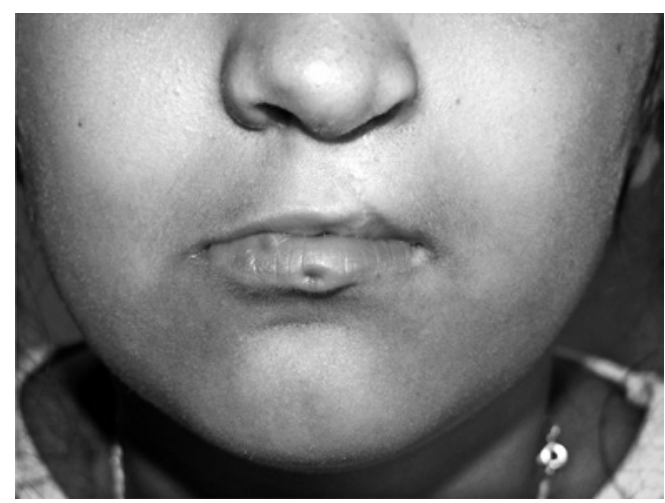

Fig. 1 B. Salivary fistula on the lower lip

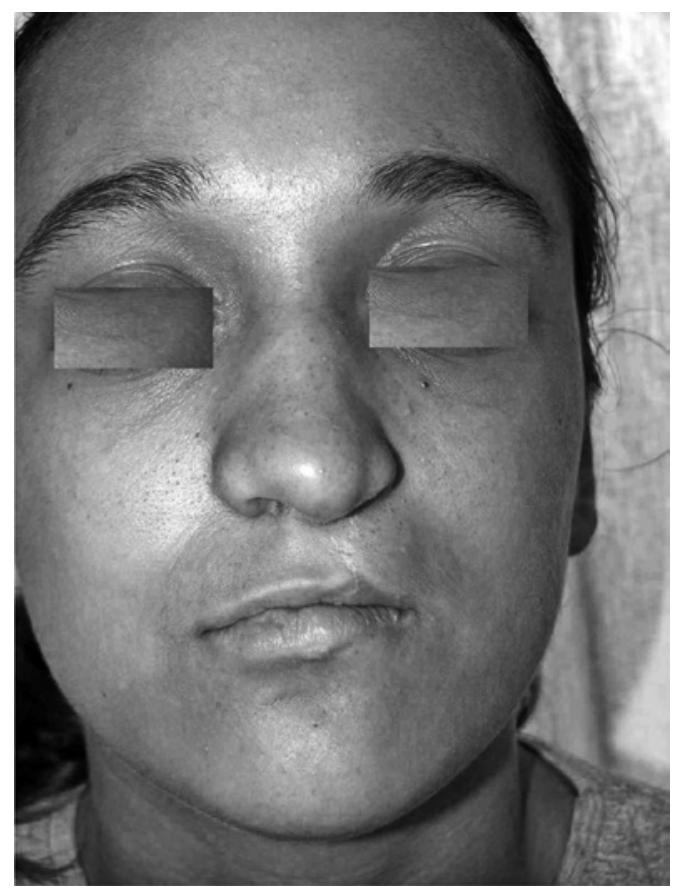

Fig. 1 C. Result after cleft surgery and salivary fistula resection illa, and short frenulum (ankyloglossia). Sometimes it coexists with heart defects, extra nipples, and Hirschsprung's disease-a type of bowel disorder [14, 15].

Van der Woude syndrome is autosomal dominant, and about $70 \%$ of patients have a mutation of transcription factor IRF6 gene (interferon regulatory factor 6). The family of IRF factors is responsible for the expression of interferon- $\alpha$ and interferon- $\beta$ during viral infection, but the function of IRF6 is not yet known [16]. The gene encoding IRF6 is located on chromosome 1 at position 1q32-q41 [17]. So far, 300 different variants of the gene encoding IRF6 have been discovered, but some are related to the phenomenon of incomplete gene penetration-there are multiple cases of healthy individuals with a mutation in IRF6 [18].

According to recent reports, VWS can also be caused by mutations in other genes. Studies on the GRHL3 gene (Grainyhead-like 3) have qualified it as a second gene responsible for a phenotype similar to Van der Woude syndrome [19]. The authors of that study hypothesize that mutations in GRHL3 are more liable to cause an isolated cleft palate than $\mathrm{CL} / \mathrm{P}$ in individuals with the IRF6 genotype. Further studies will confirm the usefulness of the diagnostics of this and other genes in predicting the probability of VWS.

IRF6 gene variants are also responsible for popliteal pterygium syndrome (PPS, OMIM 119500), which is currently considered an allelic VWS. In addition to its common phenotype (CL/P and cavities in lips), its most characteristic features are skin folds running from the heel to the popliteal area that cause contractures of the lower limbs. Other features include syndactyly, genital anomalies (e.g. cleft scrotum, hypoplasia of labia majora), and the fusion of the eyelids (ankyloblepharon). The prevalence of PPS is $1: 300000$. Research is progressing to identify mutations of other genes (e.g., RIPK4) associated with PPS [20].

\section{Ectrodactyly, Ectodermal Dysplasia, Cleft Lip and Palate (EEC3, OMIM 604292)}

The use of the term "EEC syndrome" with regard to ectrodactyly, ectodermal dysplasia, and cleft lip with/without cleft palate dates from Rüdiger's 1970 publication [21]. It describes a complex set of defects consisting of a broad, heterogeneous phenotypic group of disorders. EEC syndrome is very difficult to diagnose, because of the vari- 
ety of clinical forms that overlap with other clinical syndromes and which differ even within the same family. Ectodermal dysplasia alone, though it makes up only a part of EEC, consists of approximately 150 described anomalous structures derived from the ectoderm (thin skin that lacks sweat glands and is prone to injury, brittle hair and nails, changes in the structure and number of teeth, lacrimal duct anomalies, damage to the cornea, and so on). Cases have been described of blindness in small children suggesting a direct relationship with ectodermal anomalies in EEC [22]. Ectrodactyly is a disorder involving the partial or total absence of fingers or toes. Ectrodactyly is also referred as a "lobster claw" due to the hypoplasia of middle fingers, which often extends to the wrist, resulting in a characteristic hand shape. Cleft defects occur with varying severity, ranging from a cleft uvula, to (more frequently) a complete, wide, unilateral or bilateral cleft lip, alveolus, and palate $[23,24]$ (Fig. 2). Other less common defects include microcephaly, mental retardation, a total or partial lack of hearing, and alterations in the urogenital system [25]. EEC is a rather rare syndrome, with an estimated incidence of about $1.5: 1,000,000$ live births. To date, about 200 cases have been described [26]. Some patients affected by EEC show a form of "incomplete syndrome," lacking, for example, the ectodermal components [27].

Depending on the location of the damage to the genetic material, as well as on the phenotype of patients, we can distinguish three types of EEC syndrome. Of these, about $98 \%$ are type-3 EEC3, which is autosomal dominant and relates to the TP63 gene located on the long arm of chromosome 3 (3q28). TP63 encodes a p63-transcription factor that belongs to the p53family. To date, more than 40 distinct TP63 mutations are associated with the disorder; most are missense mutations that cause amino acid replacement in the final protein [28].

A recent case report on a Japanese girl suggested that there are other genes responsible for the occurrence of similar phenotypic manifestations [29]. In addition to the defects described in EEC, there were depressions of the lower lip characteristic of VWS and PPS. Bilateral cleft lip, alveolus, and palate were all present-a malformation common to all three syndromes. No mutations were found in studies of the TP63 gene. After a thorough analysis, the $\mathrm{R} 84 \mathrm{C}$ mutation has been identified in the IRF6 gene, as described previously for PPS. From this, it can be concluded that the phenotypic effects of IRF6 mutations can also mimic EEC syndrome.
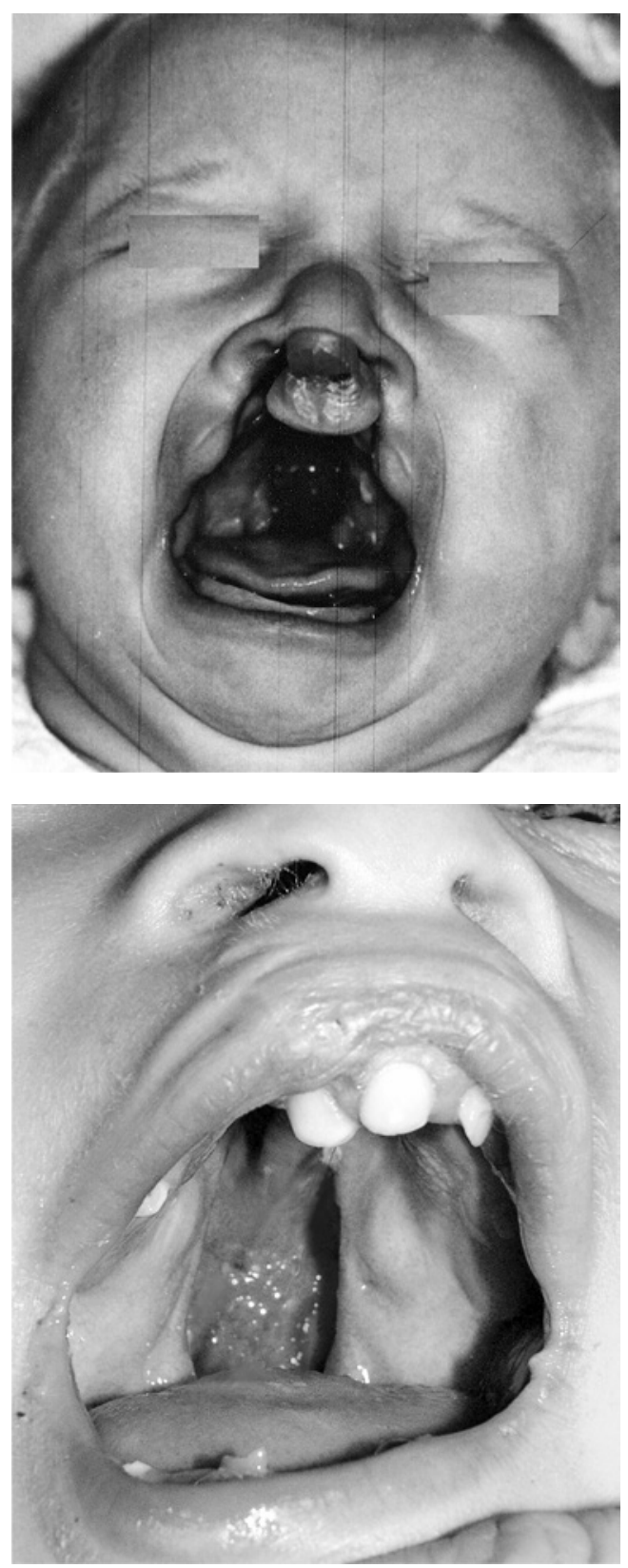

Fig. 2 A, B. The child with the ectrodactyly, ectodermal dysplasia, cleft lip, alveous and palate (EEC). Severe bilateral cleft lip, alveous and palate

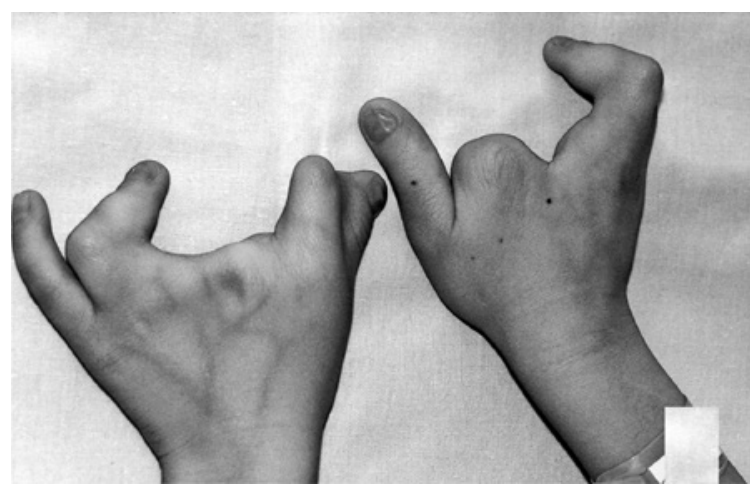

Fig. 2 C. Bilateral abnormalities of the hand (ectrodactyly) 

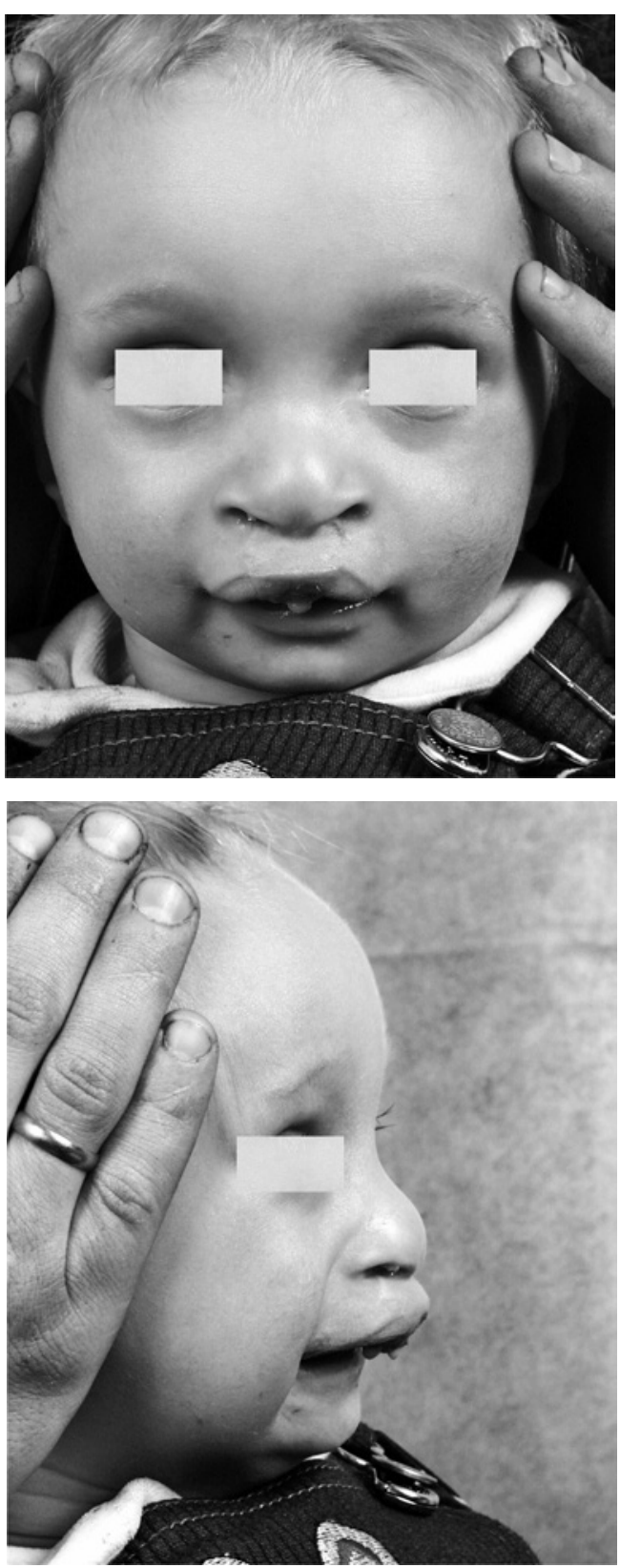

Fig. 2 D, E. Early outcome after cheiloplasty and cleft palate surgery

\section{Pierre Robin Sequence}

Pierre Robin sequence (PRS, OMIM 261800) is a condition named after the Parisian dentist who, in 1923, described the characteristic triad of deformations: The underdevelopment of the lower jaw (micrognathia), retraction of the tongue (glossoptosis), and cleft palate (Fig. 3). The prevalence of PRS is $1: 8500-1: 20000$ [30]. Impaired construction of the oral cavity and throat is responsible for difficulties in breathing, feeding, and diction. PRS is referred to as a sequence on account of its pathological mechanism in the form of a chain of developmental malformations: the occurrence of one disorder entails the next defect. It is gener-
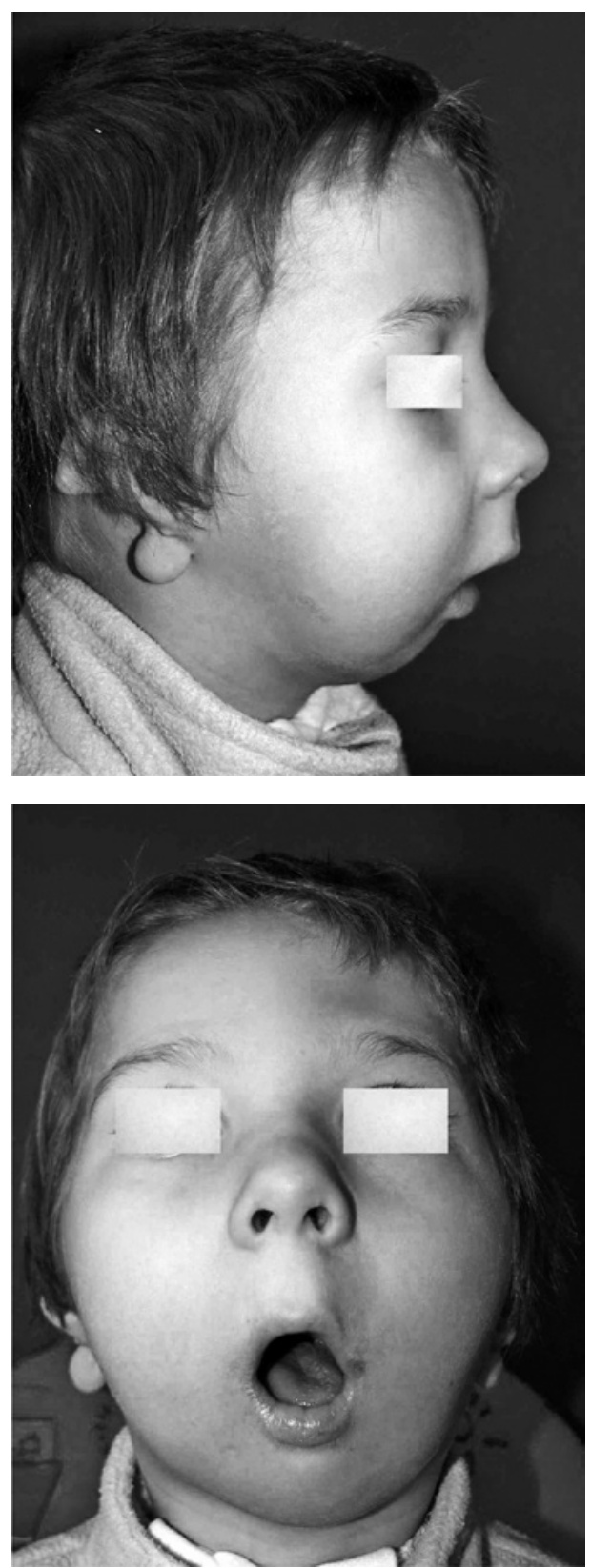

Fig. 3 A, B. The boy with Pierre Robin sequence (PRS)

ally accepted that the first defect involves the underdevelopment of the lower jaw, which interferes with the normal development of the tongue and leads to its abnormally high setting in the back of the throat. In this position, the tongue "pushes" the palatal plates and prevents their integration, resulting in a cleft palate. There are also alternative theories about the formation of PRS: one of them assumes that the primary disorder is abnormal muscle tone coupled with a deficiency of mobility of the tongue and throat, which causes the lack of a swallowing reflex - an essential element that stimulates the development of mandible length. In this case, micrognathia and cleft palate are considered the secondary defects [31]. The sequence may be an independent disorder, or may be 
part of other complex defects, such as fetal alcohol syndrome (FAS) or Stickler syndrome.

To date, several locations have been described in the human genome where a mutation can lead to PRS. Most research has focused on the region of SOX9 gene [32]. The full name of SOX9 gene is SRY (sex determining region Y)-box 9. It is located on the long arm of chromosome 17 located at position $23(17 \mathrm{q} 23)$ and is responsible for the production of a protein crucial for the normal embryonic development. The SOX9 protein is a transcription factor, which, by binding to specific DNA regions, activates other genes responsible primarily for the formation of bone and the reproductive system; it also regulates the remodeling of collagen in bone. It is remarkable that the SOX9 protein directly activates COL2A1 and COL11A2, i.e. proteins linked with the onset of Stickler syndrome [33].

Another gene that can confirm the unique role of the long arm of chromosome 17 in the development of PRS is KCNJ2. It is located very close to $S O X 9$ and encodes a potassium canal structure. According to researchers [34], damage to KCJN2 can lead to changes in the expression of the nearby SOX 9 and may contribute to a fetus developing PRS. In another study, researchers proposed a relationship between $S O X 9$ and the SATB2 gene [35]. Animal model studies suggest that the two genes work together through cis-regulation in order to coordinate the expanding growth of the mandible.

In addition, according to the recent report, the inactivation of two other genes, Ptprs and Ptprf from the leukocyte antigen-related (LAR) gene family coding receptor protein tyrosine phosphatases (RPTPs) can lead to the syndrome resembling the defects of the Pierre Robin sequence [36]. Studies of genetically modified mouse embryos have shown that Ptprs and Ptprf are necessary for the morphogenesis of the mandible. Further research on the human genome should help confirm the relationship between LAR genes and PRS.

\section{Stickler Syndrome (STL1, OMIM 108300; STL2, OMIM 604841; STL3, OMIM 184840; STL4, OMIM 614134; STL5, OMIM 614284; atypical STL, OMIM 609508)}

In 1965, Gunnar Stickler et al. first described connective tissue dysplasia in the form of hereditary progressive arthro-ophthalmopathy (AO) with threatening retinal detachment [37]. Since then, a few such disorders have been described and are collectively known as Stickler syndrome (STL). It has been demonstrated that the common origin for the formation and development of these heterogeneous diseases is determined by collagen abnormalities (particularly in collagen types II, IX and XI), which is present in most body tissues. The incidence rate of STL is estimated to be 1:7500-9000 [38]. Most types of STL involve specific disorders of the eyeball (vitreous abnormalities; congenital myopia, possibly coexisting with cataracts; and retinal damage leading to detachment). In addition to ocular disorders, STL may also involve cleft palate defects with varying degrees of severity (from cleft uvula, through submucosal cleft palate, to phenotypic variants of PRS), changes in craniofacial structure (flat face, collapsed nasal bridge), changes in joints and bones (spine anomalies, scoliosis, kyphosis, chronic joint pain, joint hypermobility that recedes with age), and progressive hearing loss (on the basis of recurrent otitis media and damage to the structures of the middle and inner ear, sensorineural hearing loss) [39].

About $75 \%$ of STL is type I (STL1). This is autosomal dominant and associated with abnormalities in type II collagen structure. Type II collagen is the main constituent of the vitreous of the eye, of cartilage, and of nucleus pulposus. Due to the characteristic image of the intraocular structures in a slit lamp, STL1 is often called the vitreous membranous type. Patients with STL1 often have also other defects, such as myopia, cleft palate, flat face, mild conductive hearing disorder, and degenerative changes in the joints occurring early (in the third or fourth decade of life). Patients with STL1 have the highest risk of retinal detachment and blindness of all types, up to 70\% [40]. The most common loss of function mutation occurs in the COL2A1 gene, located on the long arm of chromosome 12 (12q13.11) [41]. So far, about 90 different mutations of COL2A1 are known to lead to the formation of Stickler syndrome [42].

In autosomal dominant syndrome type II (STL2), mutation occurs in the gene encoding the al chain of collagen XI in the COL11A1 gene, which is located on the short arm of chromosome 1 (1p21.1). In contrast to STL1, individuals with STL2 show profound hearing loss, and some have normal vitreous; others have a beaded vitreous architecture characteristic of STL2. The risk of retinal damage is less than in type I, but is still around $40-50 \%$ [43]. In addition to the standard methods of identifying changes in DNA by RT-PCR, a unique method of identifying mutations in the COL11A1 gene using multiplex ligation-dependent probe amplification (MLPA) has 
recently been developed [44]. In a study employing this method, six new and previously unknown deletions in the $1 \mathrm{p} 21$ region were found. These might have been overlooked in the standard method of exon sequencing. This method may help us find new mutations in other types of STL.

Stickler syndrome type III (STL3), known as non-ocular Stickler syndrome, is caused by deletions of the COL11A2 gene that encodes the $\alpha 2$ chain of collagen type XI. COL11A2 is located on chromosome 6 (6p21.3). The $a 2$ chain is not present in the structure of the vitreous, hence the absence of ocular alterations in STL3. The changes that have been described in the membrane covering the organ of Corti in two families with STL3 are probably related to the high expression of this type of collagen in that area. This results in nonprogressive congenital hearing loss to a small or moderate extent, often accompanied by cleft palate and arthropathy [45].

It was for a long time believed that STL mutations were only autosomal dominant. In 2006, a case of a Moroccan family was described in which there were symptoms suggestive of STL, but there were no known mutations in collagen II or XI [46]. The family members suffered from the following disorders: short stature (between the $10^{\text {th }}$ and $25^{\text {th }}$ percentile), knee valgus, flat face, amblyopia, myopia with astigmatism, vitreous syneresis (shrinkage as a result of the progressive transformation of the gel to liquid), jaw underdevelopment, flattened metacarpal heads, sensorineural impaired hearing. None of the patients, however, had a cleft defect. After a thorough genetic analysis, a new set of genetic mutations were found to be associated with autosomal recessive disorder of the al chain structure of collagen type IX. The COL9A1 gene responsible for STL4 was found on the long arm of chromosome 6 (at 6q13). Further mutations in the COL9A1 gene have been described in 2011, in two Turkish sisters and one Moroccan male [47]. In the same year, the case of an Indian family with autosomal recessive mutation in the COL9A2 gene on chromosome 1 (1p33-32) was described with a phenotype similar to that of STL4, also lacking a cleft. The mutation was classified as Stickler syndrome type $\mathrm{V}$. The most recent discovery in the context of STL was described in January 2014 as another variant associated with autosomal recessive inheritance [48]. These alterations were discovered in three Moroccan siblings. All the children suffered from massive myopia, partial hearing loss, hypoplasia of the midface, abnormal internal rotation of the tibia, flat feet, and down-slanting palpebral fissures. These patients also presented no cleft defects. This type of STL is associated with the mutation of the gene encoding collagen IX; the mutation refers to the gene COL9A3 that encodes the $\alpha 3$ collagen chain and is located on the long arm of chromosome 20 (20q13). There are also known STL variants that include only the ocular structures and present no changes in other organs. These varieties are collectively referred to as atypical STL [49]. Further observations and genetic research is expected to reveal new varieties of this syndrome, which is very diverse in phenotype and genotype.

\section{Treacher Collins Syndrome (TCS1, OMIM 154500; TCS2, OMIM 613717; TCS3, OMIM 248390)}

This syndrome of defects which take the form of mandibulofacial dysostosis was first mentioned by George Andreas Berry in 1889, and was well described in 1900 by the English ophthalmologist Edward Treacher Collins, after whom it is now named [50]. In German-speaking countries, TCS is also called Treacher Collins-Franceschetti syndrome or Franceschetti-Klein syndrome, after the Swiss researchers who systematized the available knowledge of TCS in 1949 and classified it as a dysostosis [51]. TCS also belongs to the Tessier classification of rare facial clefts as cleft number 6-78 [52]. The syndrome occurs with a frequency of $1: 50000$, and is connected to the development of the structures derived from the first and second pharyngeal arches [53]. Deformations occur on both sides, but bilateral facial symmetry is not present. The basic features of TCS include changes around the eye sockets, ears, zygomatic bone, maxilla, and mandible. Patients with TCS usually have down-slanting palpebral fissures with weakened orbicularis oculi muscles and a characteristic lack of eyelashes in the central third of the lower eyelid [54]. An important element of the syndrome is a variously expressed coloboma - a cleft of the eyelid and eye structures. In some patients, there are tear ducts and lacrimal puncta atresia. The majority of patients have distortion of hearing to varying degrees caused by many anatomical defects, from bilateral hypoplasia and aplasia of the pinna (microtia and anotia), through stenosis and atresia of the external auditory canal, tympanic membrane deformation, and abnormal ossification of the ear ossicles, to completely undeveloped middle-ear structures. For this reason, such patients have partial or total deafness, despite possessing well-developed inner ear structures. A constant element of TCS is hypoplasia of the zygomatic and temporal bones with occasional ankylosis of the temporomandibular joint, micrognathia with retrognathia, 

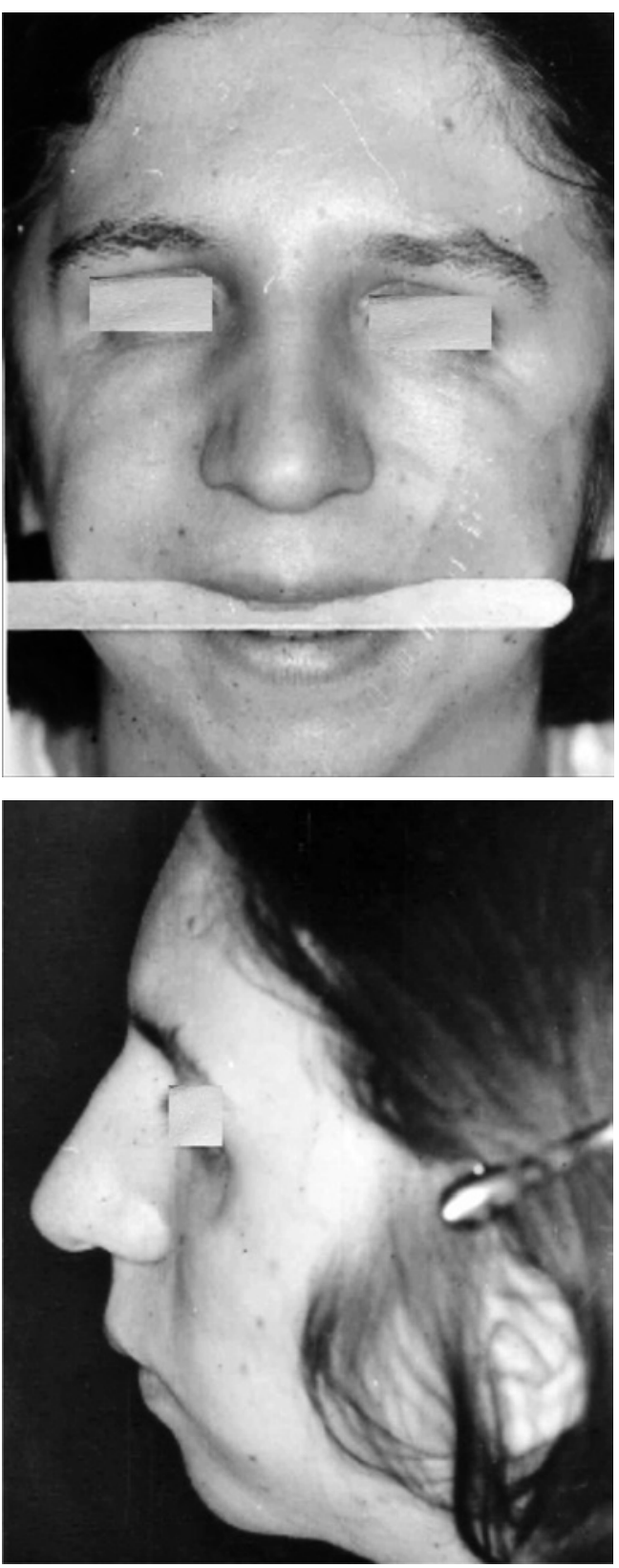

Fig. 4. A, B. The girl with Treacher Collins syndrome (TCS) - a down-slanting palpebral fissures, deformation of the lower and lateral orbital rim

a large and protruding nose, which gives the patients a characteristic "bird-like" facial appearance (Fig. 4). In about $30 \%$ of cases, there is submucosal, partial, or complete cleft palate with cleft lip. Occasionally, it coexists with choanal atresia, aplasia of the parotid gland, defects of the cervical spine, kidneys, cryptorchidism, and heart defects [55].

TCS is phenotypically very diverse, even within the same genomic disorder. There are three types of Treacher Collins syndrome, depending on the genetic background. More than $90 \%$ of TCS is TCS1, caused by an autosomal dominant mutation of the TCOF1 gene (Treacher Collins-Franceschetti syndrome 1), are located on the long arm of chromosome 5 (5q32) [56]. About $60 \%$ are
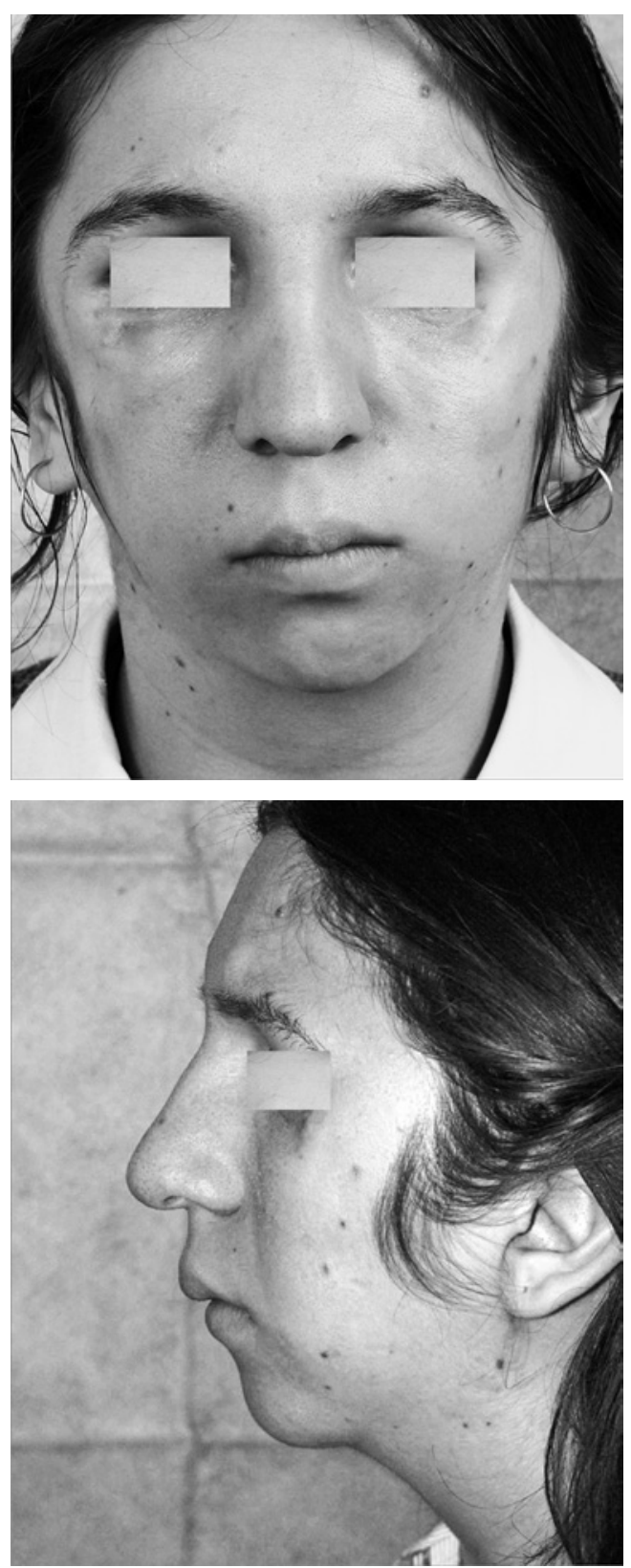

Fig. 4. C, D. Early outcome after orbital rim reconstruction with bone graft

de novo mutations, with the remaining $40 \%$ mutations inherited from parents. To date, more than 120 TCOF1 gene mutations leading to TCS have been described, with further disturbances revealed in recent years [57]. These are mostly mutations connected with the loss of function. The product of the TCOF1 gene is treacle protein, which is involved in the formation of ribosomes as a regulator of ribosomal DNA gene transcription. According to recent studies, the pathogenesis of TCS is also related to p53: mutation in one allele of the TCOF1 gene induces cellular stress that activates p53, which leads to death and abnormal differentiation of neuroepithelial cells [58]. An animal model of TCS with a TCOF1-equivalent gene has al- 
so been developed. Studies on freshwater zebrafish (Danio rerio) have proven that artificial disruption of B8JIY2 gene expression (a TCOF1 ortholog in this species) allows for the formation of individuals with phenotypes resembling Treacher Collins syndrome [59]. Further use of this model should demonstrate the exact role of TCOF1 in the formation of TCS. The other studies of TCS1 etiology that have been carried out on the human genome have revealed that not only single mutations are responsible for the TCS; it can also occur on the basis of the total rearrangement of the TCOF1 gene [60].

In some cases, however, no signs of gene mutations are found to be associated with TCOF1, which raises suspicions of the existence of pathologies in other genes that lead to the formation of this syndrome. This was confirmed by the results of studies published in 2011. The mutations in POLR1D and POLR1C genes were described as being phenotypically identical to TCS [61]. Both genes are responsible for encoding the proteins that form the subunits of RNA polymerase I and III. These mutations cause a reduction in the number of ribosomes in neuroepithelial cells, thus confirming the etiology of TCS as a ribosomopathy. The POLR1D gene is located on the long arm of chromosome 13 (13q12) and is associated with the autosomal dominant type- 2 syndrome, TCS2. The same report describes an entirely new type of inheritance previously unknown for TCS. Three patients had mutations in both POLR1C alleles located on the short arm of chromosome 6 (6p21), operatively associated with POLR1D. Studies carried out on the carrier parents have confirmed for the first time the autosomal recessive etiology of the disease and, as a result, type-3 Treacher Collins syndrome was isolated (TCS3). In the report from September 2014, autosomal recessive POLR1D gene mutations were also discovered [62]. Future studies on the ribosomal biogenesis pathway should identify additional ribosomopathies phenotypically associated with TCS.

\section{Summary}

Complex congenital malformations involving cleft anomalies are clinically a very diverse group. For this reason, they require close cooperation of doctors from almost all specialties. The individual syndromes have different incidences and most of them also have their own allelic variants, with the characteristic severity of the anomaly differing even across close relatives. Although several thousand different mutations responsible for the syndromes have been discovered so far, there are many phenotypic cases of unknown genetic cause. Easier access to genetic counseling and lower costs of DNA testing have led to new findings regarding the causes of the syndromes. This trend will certainly continue in the coming years, which will allow for more accurate diagnosis and early treatment of children suffering from these diseases.

\section{References}

[1] Bender PL: Genetics of cleft lip and palate. J Pediatr Nurs 2000, 15, 242-249.

[2] Murray JC: Gene/environment causes of cleft lip and/or palate. Clin Genet 2002, 61, 248-256.

[3] Dixon MJ, Marazita ML, Beaty TH: Cleft lip and palate: Understanding genetic and environmental influences. Nat Rev Genet 2011, 12, 167-178.

[4] Fitzpatrick D, Farrall M: An estimation of the number of susceptibility loci for isolated cleft palate. J Craniofac Genet Dev Biol 1993, 13, 230-235.

[5] Boo-Chai K. An ancient Chinese text on a cleft lip. Plast Reconstr Surg 1966, 38, 89-91.

[6] von Graefe CF: Bericht über die erste Staphyloplastick. Hufeland's J 1817, 44, 116.

[7] von Langenbeck G: Operation der angeborenen totalen Spaltung des harten Gaumens nach einer neuen Methode. Deutsche Klin 1861, 8, 231-232.

[8] Veau, V: La division palatine. Paris: Masson 1931.

[9] Johnson CY, Little J: Folate intake, markers of folate status and oral clefts: Is the evidence converging? Int J Epidemiol 2008, 37, 1041-1058.

[10] Johansen AM, Lie RT, Wilcox AJ: Maternal dietary intake of vitamin A and risk of orofacial clefts: A populationbased case-control study in Norway. Am J Epidemiol 2008, 167, 1164-1170.

[11] McKinney CM, Chowchuen B, Pitiphat W: Micronutrients and oral clefts: A case-control study. J Dent Res 2013, 92, 1089-1094.

[12] Wang W, Guan P, Xu W: Risk factors for oral clefts: A population-based case-control study in Shenyang, China. Paediatr Perinat Epidemiol 2009, 23, 310-320.

[13] Van der Woude A: Fistula labii inferioris congenita and its association with cleft lip and palate. Am J Hum Genet $1954,6,244-256$.

[14] Rizos M, Spyropoulos MN: Van der Woude syndrome: A review. Cardinal signs, epidemiology, associated features, differential diagnosis, expressivity, genetic counselling and treatment. Eur J Orthod 2004, 26, 17-24.

[15] Wójcicki P, Kobus K, Wójcicka K: Van der Woude syndrome. Dent Med Probl 2007, 44, 18-21.

[16] Mostowska A, Wójcicki P, Kobus K: Gene symbol: IRF6. Disease: Van der Woude syndrome. Hum Genet 2005, $116,534$. 
[17] Kondo S, Schutte BC, Richardson RJ: Mutations in IRF6 cause Van der Woude and popliteal pterygium syndromes. Nat Genet 2002, 32, 285-289.

[18] Leslie EJ, Standley J, Compton J: Comparative analysis of IRF6 variants in families with Van der Woude syndrome and popliteal pterygium syndrome using public whole-exome databases. Genet Med 2013, 15, 338-344.

[19] Peyrard-Janvid M, Leslie EJ, Kousa YA: Dominant mutations in GRHL3 cause Van der Woude Syndrome and disrupt oral periderm development. Am J Hum Genet 2014, 94, 23-32.

[20] Qasim M, Shaukat M: Popliteal pterygium syndrome: A rare entity. APSP J Case Rep 2012, 3, 5.

[21] Rüdiger RA, Haase W, Passarge E: Association of ectrodactyly, ectodermal dysplasia, and cleft lip-palate. Am J Dis Child 1970, 120, 160-163.

[22] Rosenberg JB, Butrus S, Bazemore MG: Ectrodactyly-ectodermal dysplasia-clefting syndrome causing blindness in a child. J AAPOS 2011, 15, 80-82.

[23] Trope BM, Salomão JN, Costa VD: Do you know this syndrome? Ectrodactyly - ectodermal dysplasia - cleft lip/ palate (EEC) syndrome. An Bras Dermatol 2010, 85, 573-575.

[24] Wójcicki P, Wysocki M, Wójcicka K: Ectrodactyly-ectodermal dysplasia-clefting syndrome-plastic surgeon's considerations. J Craniofac Surg 2010, 21, 1388-1392.

[25] Rollnick BR, Hoo JJ: Genitourinary anomalies are a component manifestation in the ectodermal dysplasia, ectrodactyly, cleft lip/palate (EEC) syndrome. Am J Med Genet 1988, 29, 131-136.

[26] Marwaha M, Nanda KD: Ectrodactyly, ectodermal dysplasia, cleft lip, and palate (EEC syndrome). Contemp Clin Dent 2012, 3, 205-208.

[27] Shivaprakash PK, Joshi HV, Noorani H: Ectrodactyly, ectodermal dysplasia, and cleft lip/palate syndrome: A case report of "Incomplete syndrome". Contemp Clin Dent 2012, 3, Suppl 1, 115-117.

[28] Barbaro V, Confalonieri L, Vallini I: Development of an allele-specific real-time PCR assay for discrimination and quantification of p63 R2 79H mutation in EEC syndrome. J Mol Diagn 2012, 14, 38-45.

[29] Kosaki R, Kaneko T, Torii C, Kosaki K: EEC syndrome-like phenotype in a patient with an IRF6 mutation. Am J Med Genet, Part A, 2012, 158A, 1219-1220.

[30] Breugem CC, Mink van der Molen AB: What is 'Pierre Robin sequence'? J Plast Reconstr Aesthet Surg 2009, 62, 1555-1558.

[31] Sherer DM, Metlay LA, Woods JR Jr: Lack of mandibular movement manifested by absent fetal swallowing: A possible factor in the pathogenesis of micrognathia. Am J Perinatol 1995 12, 30-33.

[32] Selvi R, Mukunda Priyanka A: Role of SOX9 in the etiology of Pierre-Robin Syndrome. Iran J Basic Med Sci 2013, $16,700-704$.

[33] Bridgewater LC, Lefebvre V, de Crombrugghe B: Chondrocyte-specific enhancer elements in the Col11a2 gene resemble the Col2a1 tissue-specific enhancer. J Biol Chem 1998, 273, 14998-15006.

[34] Jakobsen LP, Ullmann R, Christensen SB: Pierre Robin sequence may be caused by dysregulation of SOX9 and KCNJ2. J Med Genet 2007, 44, 381-386.

[35] Rainger JK, Bhatia S, Bengani H: Disruption of SATB2 or its long-range cis-regulation by SOX9 causes a syndromic form of Pierre Robin sequence. Hum Mol Genet 2014 23, 2569-2579.

[36] Stewart K, Uetani N, Hendriks W: Inactivation of LAR family phosphatase genes Ptprs and Ptprf causes craniofacial malformations resembling Pierre-Robin sequence. Development 2013, 140, 3413-3422.

[37] Stickler GB, Belau PG, Farrell FJ: Hereditary progressive arthro-ophthalmopathy. Mayo Clin Proc 1965, 40, 433-455.

[38] Hoornaert KP, Vereecke I, Dewinter C: Stickler syndrome caused by COL2A1 mutations: Genotype-phenotype correlation in a series of 100 patients. Eur J Hum Genet 2010, 18, 872-880.

[39] Snead MP, McNinch AM, Poulson AV: Stickler syndrome, ocular-only variants and a key diagnostic role for the ophthalmologist. Eye (Lond) 2011, 25, 1389-1400.

[40] Ang A, Poulson AV, Goodburn SF: Retinal detachment and prophylaxis in type 1 Stickler syndrome. Ophthalmology 2008, 115, 164-168.

[41] Parke DW: Stickler syndrome: Clinical care and molecular genetics. Am J Ophthalmol 2002, 134, 746-748.

[42] Zechi-Ceide RM, Jesus Oliveira NA, Guion-Almeida ML: Clinical evaluation and COL2A1 gene analysis in 21 Brazilian families with Stickler syndrome: Identification of novel mutations, further genotype/phenotype correlation, and its implications for the diagnosis. Eur J Med Genet 2008, 51, 183-196.

[43] Poulson AV, Hooymans JM, Richards AJ: Clinical features of type 2 Stickler syndrome. J Med Genet 2004, 41, e107.

[44] Vijzelaar R, Waller S, Errami A: Deletions within COL11A1 in Type 2 stickler syndrome detected by multiplex ligation-dependent probe amplification (MLPA). BMC Med Genet 2013, 14, 48.

[45] van Beelen E, Leijendeckers JM, Huygen PL: Audiometric characteristics of two Dutch families with non-ocular Stickler syndrome (COL11A2). Hear Res 2012, 291, 15-23.

[46] Van Camp G, Snoeckx RL, Hilgert N: A new autosomal recessive form of Stickler syndrome is caused by a mutation in the COL9A1 gene. Am J Hum Genet 2006, 79, 449-457.

[47] Nikopoulos K, Schrauwen I, Simon M: Autosomal recessive Stickler syndrome in two families is caused by mutations in the COL9A1 gene. Invest Ophthalmol Vis Sci 2011, 52, 4774-4779.

[48] Faletra F, D’Adamo AP, Bruno I: Autosomal recessive Stickler syndrome due to a loss of function mutation in the COL9A3 gene. Am J Med Genet A 2014, 164A, 42-47.

[49] Richards AJ, Martin S, Yates JR: COL2A1 exon 2 mutations: Relevance to the Stickler and Wagner syndromes. Br J Ophthalmol 2000, 84, 364-371. 
[50] Treacher Collis E: Cases with symmetrical congenital notches in the outer part of each lid and defective development of the malar bones. Trans Ophthalmol Soc UK 1900, 20, 190-192.

[51] Franceschetti A, Klein D: Mandibulo-facial dysostosis: New hereditary syndrome. Acta Ophtalmol 1949, $27,143-224$.

[52] Tessier P: Anatomical classification facial, cranio-facial and latero-facial clefts. J Maxillofac Surg 1976, 4, 69-92.

[53] Chang CC, Steinbacher DM: Treacher Collins syndrome. Semin Plast Surg 2012, 26, 83-90.

[54] Kobus K, Wójcicki P: Surgical treatment of Treacher Collins syndrome. Ann Plast Surg 2006, 56, 549-554.

[55] Wójcicki P, Marszałek-Kruk B: Uwarunkowania genetyczne oraz zasady leczenia zespołu Treachera Collinsa. Dent Med Probl 2005, 42, 619-626.

[56] Kadakia S, Helman SN, Badhey AK: Treacher Collins Syndrome: The genetics of a craniofacial disease. Int J Pediatr Otorhinolaryngol 2014, 78, 893-898.

[57] Marszałek-Kruk BA, Wójcicki P, Śmigiel R: Novel insertion in exon 5 of the TCOF1 gene in twin sisters with Treacher Collins syndrome. J Appl Genet 2012, 53, 279-282.

[58] Sloan KE, Bohnsack MT, Watkins NJ: The 5S RNP couples p53 homeostasis to ribosome biogenesis and nucleolar stress. Cell Rep 2013, 5, 237-247.

[59] Weiner AM, Scampoli NL, Calcaterra NB: Fishing the molecular bases of Treacher Collins syndrome. PLoS One 2012, 7, e29574.

[60] Bowman M, Oldridge M, Archer C: Gross deletions in TCOF1 are a cause of Treacher-Collins-Franceschetti syndrome. Eur J Hum Genet 2012, 20, 769-777.

[61] Dauwerse JG, Dixon J, Seland S: Mutations in genes encoding subunits of RNA polymerases I and III cause Treacher Collins syndrome. Nat Genet 2011, 43, 20-22.

[62] Schaefer E, Collet C, Genevieve D: Autosomal recessive POLR1D mutation with decrease of TCOF1 mRNA is responsible for Treacher Collins syndrome. Genet Med 2014, 16, 720-724.

\section{Address for correspondence:}

Maciej Koźlik

Department of Plastic Surgery

Wroclaw Medical University

ul. Jana Pawła II 2

57-320 Polanica-Zdrój

Poland

E-mail: maciej.kozlik@gmail.com

Conflict of interest: None declared

Received: 15.11.2015

Revised: 19.12.2015

Accepted: 24.02.2016 